\title{
Metabolic and endocrine manifestations of neoplastic disease
}

\section{Introduction}

Neoplastic diseases, both benign and malignant, can present as a variety of metabolic and endocrine manifestations. They range from a single biochemical abnormality to a full-blown endocrine syndrome and often precede clinical detection of the tumour, providing useful clues for diagnosis.

\section{Pathophysiology}

Different mechanisms underlie metabolic and endocrine derangements caused by neoplasia. The most common mechanism involves invasion of an endocrine organ by the neoplasm. This often leads to a state of hormone deficiency. Hypogonadism caused by craniopharyngioma, and a hypoadrenal state caused by lymphoma are two examples of this category.

A second mechanism involves synthesis of substances with hormonal activity by neoplastic cells. This category includes the classical paraneoplastic syndromes and the causative tumour is often located in non-endocrine organs, hence they are called ectopic hormone secreting tumours. The mechanism by which neoplastic cells of non-endocrine organs acquire hormone secreting ability is obscure. The process of "de-repression" of genes that accompany malignant transformation of cells has been incriminated.

Most of the metabolically active substances produced by tumours are peptides. Some of them are structurally similar to the original hormone eg. parathyroid hormone related protein (PTH-r-P). But most are structurally different from the original hormone eg. insulin like growth factors (IGF - I and II) secreted by the nonislet cell tumours. Steroid hormones, which require complex enzymatic pathways for synthesis, are rarely synthesized by ectopic tumours.

Because neoplastic cells lack receptors, and their autonomous secretion of hormone-like substances, tropic hormones or physiological stimuli cannot exert any effect on the rate of ectopic hormone secretion. Conventional suppression tests used in the diagnosis of hormone excess cannot suppress elevated level of hormone-like substances produced by neoplasms. Techniques used for estimation of hormones often cannot detect these substances because of their structural differences from the natural hormone.

The third mechanism involves synthesis of excess hormone by tumours arising within endocrine organs. Except for rare occasions, these tumours are benign and small. Sometimes such tumours involve multiple endocrine organs. Multiple endocrine neoplasia (MEN) which involves tumours in the pituitary, pancreas, parathyroid, and adrenal glands is one example.

This article reviews the pathogenic mechanisms of common and clinically relevant endocrine and metabolic manifestations of neoplasia.

\section{Hypercalcaemia}

Hypercalcaemia is the commonest metabolic abnormality observed in neoplastic disease, occurring in nearly $5 \%$ of all cancers. Malignant tumours are the commonest cause for hypercalcaemia in hospital practice but some benign lesions are also associated with it.

Hypercalcaemia in neoplasia (HCN) could be caused by two different mechanisms. Secretion of peptides like PTH-r-P by the tumour cells accounts for $80 \%$ of cases and secretion of substances such as interleukin II, and tumour necrosis factor accounts for the rest.

Malignancies in the lungs and breast and multiple myeloma account for $50 \%$ of cases of HCN. Less common causes include renal and prostate cancer. Rarely lymphomas and leukaemias are associated with HCN. Physical examination and a chest xray would detect the causative neoplastic lesion in more than $90 \%$ of cases of 
HCN. Hypercalcaemia is a late feature in most malignancies with the exceptions of multiple myeloma and some breast cancers, where it is seen as an early feature.

Primary hyperparathyroidism $(\mathrm{PH})$ is also a common cause of hypercalcaemia in hospital practice. It is often caused by benign parathyroid adenomas. In contrast to $\mathrm{PH}$, hypercalcaemia caused by malignant tumours is characterised by low levels of parathyroid hormone (PTH) and 1-25 $[\mathrm{OH}]_{2}$ cholecalciferol. Patients with $\mathrm{PH}$ tend to be hyperchloraemic and mildly acidotic. Mild alkalosis with a normal chloride level is seen in malignant tumours associated hypercalcaemia. Serum phosphate level is reduced in both conditions. Malignancy associated hypercalcaemia is steroid sensitive and a $50 \%$ reduction of serum calcium level with intravenous hydrocortisone is a useful test in differentiating it from other causes of hypercalcaemia.

\section{Hyponatraemia}

Hyponatraemia is the second commonest metabolic abnormality associated with neoplasia. Small cell carcinoma of lung (SCLC) is the commonest underlying tumour with $10-15 \%$ of patients having hyponatraemia at the time of diagnosis. Hyponatraemia is also reported in tumours arising from the head and neck region, uterine cervix, prostate, brain and in carcinoid tumours. Hyponatraemia is a component of syndrome of inappropriate antidiuretic hormone secretion (SIADH) and is due to ectopic secretion of antidiuretic hormone (ADH) or substances with similar activity by tumour cells.

Laboratory investigations are not very helpful in the differentiation of tumour associated hyponatraemia from other causes, and search for a causative tumour should be arranged in unexplained hyponatraemia.

\section{Cushing syndrome}

Cushing syndrome (CS) is rare, but the commonest tumour associated endocrine disease. Endogenous CS is caused by excess secretion of corticotrophin by a pituitary adenoma (Cushing disease) in $80 \%$, substances with corticotrophin like activity secreted by paraneoplastic tumours in $15 \%$, and by cortisol secreting adrenal tumours in $5 \%$. SCLC account for about $75 \%$ of paraneoplastic tumour associated CS.

Several clinical and biochemical features help in the differentiation of an underlying cause for CS. Cushing disease is characterised by a slowly progressive course. The elevated cortisol in such patients can be suppressed by the high dose dexamethasone suppression test. In contrast, CS due to paraneoplastic tumours causes a rapidly progressive course and the elevated cortisol level cannot be suppressed with high dose dexamathasone test. Other characteristic features of CS due to paraneoplastic syndrome include severe hypokalaemia, metabolic alkalosis, marked skin pigmentation and proximal myopathy. The ACTH level is very low in CS due to adrenal tumours and could be markedly elevated in CS due to ectopic tumours.

\section{Hypoglycaemia}

Insulin secreting neoplastic growths of pancreatic islet cells (insulinomas) and a variety of slow-growing, bulky, non-pancreatic neoplasms are associated with hypoglycaemia. Fibrosarcomas, rhabdomyosarcomas, mesotheliomas, hemangiopericytomas, hepatocellular carcinomas, lymphomas, and adrenal tumours account for the rest. One third of the non-islet cell tumours causing hypoglycaemia are retroperitoneal.

Insulin-like growth factors (IGF) secreted by these tumours lead to hypoglycaemia by different mechanisms involving increased uptake of glucose by tumour cells and by peripheral tissues and by reducing hepatic glucose output.

Differentiation of hypoglycaemia associated with neoplastic disease is based on assessment of fasting insulin and c-peptide levels. In islet cell neoplasms, fasting hypoglycaemia is accompanied by high levels of both fasting insulin and c-peptide levels. But in non-islet cell neoplasia, low levels of insulin and c-peptide are characteristic. Increased cortisol and glucagon levels with a reduced level of growth hormone is also reported in hypoglycaemia due to non-islet tissue neoplasia. Characteristically, most islet cell neoplasia cause weight gain but weight loss is a dominant feature in non-islet cell tumour associated hypoglycaemia.

\section{Pitiutary dysfunction}

Neoplastic growths within and adjacent to the pituitary gland can be associated with a number of endocrine abnormalities resulting from an excess or deficiency of one or more pituitary hormones.

Adenomas of a single type of cell cause an excess of a one hormone, and prolactin secreting adenomas are the commonest. In women these tumours are often less than $10 \mathrm{~mm}$ in size (micro-prolactinomas), and larger tumours (macro-prolactinomas $>10 \mathrm{~mm}$ ) occur in men. The clinical features in women include galactorrhoea, menstrual irregularities and subfertility, and in men they cause hypogonadism, erectile dysfunction, and oligospermia.

Acromegaly caused by somatotrophic adenomas producing growth hormone and Cushing disease caused by adenomas of corticotrophin cells are the other two endocrine manifestations of pituitary tumours. Both somatotrophic and corticotrophic adenomas are slow growing and the clinical syndrome precedes the detection of local tumour. Unlike in Cushing disease, where the pituitary fossa is often normal on a skull xray, an enlarged pituitary fossa is seen in more than $50 \%$ at the time of clinical diagnosis of acromegaly. 
Non-functioning pituitary tumours and neoplastic growths from adjacent structures such as craniopharyngiomas can cause deficiency of pituitary hormones by pressure. Clinical features vary from a deficiency of a single hormone to a syndrome of hypopitiutarism characterised by hypogonadism, growth retardation, hypoadrenocorticism and hypothyroidism. These tumours are common in childhood and middle age.

Haemorrhage into a primary pituitary tumour or to metastatic deposits can cause sudden increase in intracranial pressure, and an emergency state of acute hypopitiutarism (pituitary apoplexy). Sudden severe headache, vomiting, neck stiffness and hypotension (due to acute secondary adrenocorticism) are the features of pituitary apoplexy.

\section{Hypoadrenalism}

Primary tumours and metastases invading the adrenal glands can lead to both acute and chronic hypoadrenalism. Acute hypoadrenalism is a medical emergency characterised by severe hypotension, and the chronic hypoadrenal state is indistinguishable from other hypoadrenal states such as Addison disease.

\section{Miscellaneous abnormalities}

Isolated metabolic derangements such as hyperuricaemia and hyperkalaemia are reported with haematological malignancies. Early recognition and appropriate intervention play an important role in the management of these conditions.

\section{Further reading}

1. Lucy Y, Dirix A, Van Oosteron. Metabolic complications. In: Robert LS, Ian T, Peter H, Jean CH, eds. Oxford Textbook of Oncology v 1. New York: Oxford University Press, 2002: 897-932.

2. John WJ, Foon KA, Patchel RA. Paraneoplastic syndromes. In: Devita VT, Holamann S, eds. Principles and Practice of Oncology 5th edition. Philadelphia: Lipponcot Raven, 1992: 2397-422.

3. Mundy GR, Guise AT. Hypercalcemia of malignancy. American Journal of Medicine 1997;103:134-45.

4. Chute JP, Taylor E, Williams J, Kaye F, Venzon D, Johnson BE. A metabolic study of patients with lung cancer and hyponatraemia of malignancy. Clinics in Cancer Research 2006; 12: 888-96.

5. Young WF. The incidentally discovered adrenal mass. The New England Journal of Medicine 2007; 356: 601-10.

6. Tucker ON, Crotty PL, Conlon KC. The management of insulinoma. British Journal of Surgery 2006; 93: 264-75.

T P Weerarathna, Department of Medicine, Faculty of Medicine, University of Ruhuna, Sri Lanka.

Correspondence: TPW, e-mail: < thilakpw@yahoo.com>. Competing interests: none declared. 